\section{The bigger picture}

\section{Tamas Vicsek}

$\mathrm{f}$ a concept is not well defined, it can be abused. This is particularly true of complexity, an inherently interdisciplinary concept that has penetrated a range of intellectual fields from physics to linguistics, but with no underlying, unified theory. Complexity has become a popular buzzword that is used in the hope of gaining attention or funding - institutes and research networks associated with complex systems grow like mushrooms.

Why and how did this vague notion become such a central motif in modern science? Is it only a fashion, a kind of sociological phenomenon, or is it a sign of a changing paradigm of our perception of the laws of nature and of the approaches required to understand them? Because almost every real system is inherently complicated, to say that a system is complex is almost an empty statement couldn't an Institute for Complex Systems just as well be called an Institute for Almost Everything? Despite these valid concerns, the world is indeed made of many highly interconnected parts on many scales, the interactions of which result in a complex behaviour that requires separate interpretations of each level. This realization forces us to appreciate the fact that new features emerge as one moves from one scale to another, so it follows that the science of complexity is about revealing the principles that govern the ways in which these new properties appear.

In the past, mankind has learned to

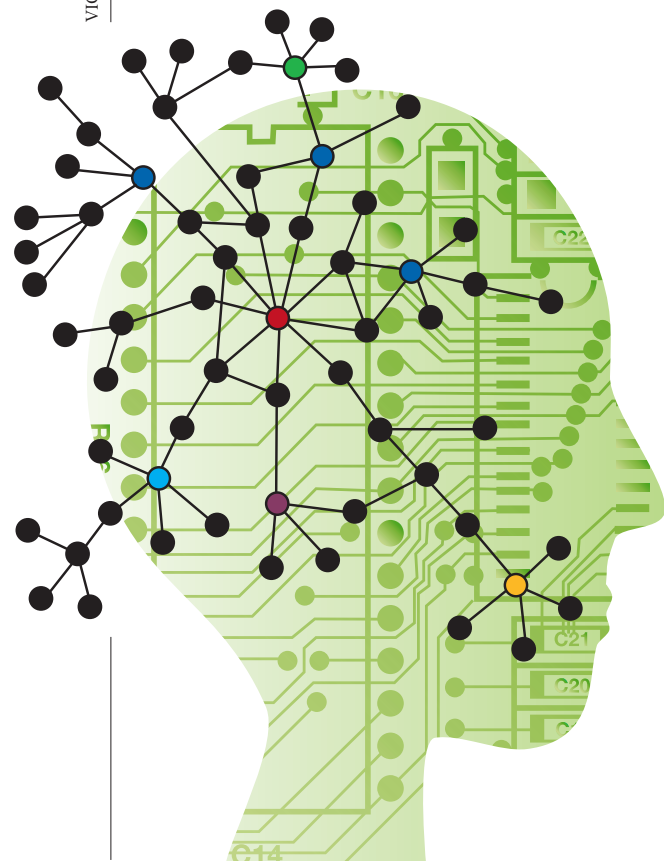

understand reality through simplification and analysis. Some important simple systems are successful idealizations or primitive models of particular real situations - for example, a perfect sphere rolling down an absolutely smooth slope in a vacuum. This is the world of newtonian mechanics, and it ignores a huge number of other, simultaneously acting factors. Although it might sometimes not matter that details such as the motions of the billions of atoms dancing inside the sphere's material are ignored, in other cases reductionism may lead to incorrect conclusions. In complex systems, we accept that processes that occur simultaneously on different scales or levels are important, and the intricate behaviour of the whole system depends on its units in a nontrivial way. Here, the description of the entire system's behaviour requires a qualitatively new theory, because the laws that describe its behaviour are qualitatively different from those that govern its individual units.

Take, for example, turbulent flows and the brain. Clearly, these are very different systems, but they share a few remarkable features, including the impossibility of predicting the rich behaviour of the whole by merely extrapolating from the behaviour of its units. Who can tell, from studying a tiny drop or a single neuron, what laws describe the intricate flow patterns in turbulence or the patterns of electrical activity produced by the brain? Moreover, in both of these systems (and in many others), randomness and determinism are both relevant to the system's overall behaviour. Such systems exist on the edge of chaos - they may exhibit almost regular behaviour, but also can change dramatically and stochastically in time and/or space as a result of small changes in conditions. This seems to be a general property of systems that are capable of producing interesting (complex) behaviour.

Knowledge of the physics of elementary particles is therefore useless for interpreting behaviour on larger scales. Each new level or scale is characterized by new, emergent laws that govern it. When creating life, nature acknowledged the existence of these levels by spontaneously separating them into molecules, macromolecules, cells, organisms, species and societies. The big question is whether there is a unified theory for the ways in which elements of a system organize themselves to produce a behaviour that is typical of large classes of systems.

Interesting principles have been proposed in an attempt to provide such a unified theory. These include self-organization, simultaneous existence of many degrees of freedom, self-adaptation, rugged energy landscapes,

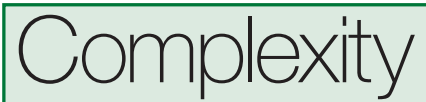

The laws that describe the

behaviour of a complex system are

qualitatively different from those that

govern its units.

and scaling (for example, power-law dependence) of the parameters and the underlying network of connections. Physicists are learning how to build relatively simple models that can produce complicated behaviour, whereas those who work on inherently very complex systems (such as biologists and economists) are uncovering ways to interpret their subjects in terms of interacting, well-defined units (such as proteins).

What we are witnessing in this context is a change of paradigm in attempts to understand our world as we realize that the laws of the whole cannot be deduced by digging deeper into the details. In a way, this change has been wrought by the development of instruments. Traditionally, improved microscopes or bigger telescopes are built to gain a better understanding of particular problems. But computers have allowed new ways of learning. By directly modelling a system made of many units, one can observe, manipulate and understand the behaviour of the whole system much better than before, as in the cases of networks of model neurons and virtual auctions by intelligent agents, for example. In this sense, a computer is a tool that improves not our sight (as does the microscope or telescope), but rather our insight into mechanisms within complex systems.

Many scientists implicitly assume that we understand a particular phenomenon if we have a (computer) model that provides results that are consistent with observations and that makes correct predictions. Yet such models make it possible to simulate systems that are far more complex than the simplest newtonian ones that allow deterministic, accurate predictions of future events. In contrast, models of complex systems frequently result in a new conceptual interpretation of the behaviour. The aim is to capture the principal laws behind the exciting variety of new phenomena that become apparent when the many units of a complex system interact. Tamas Vicsek is in the Department of Biological Physics, Eötvös University, Budapest,

Pázmàny Stny 1A, H-1117 Hungary.

\section{FURTHER READING}

Waldrop, M. M. Complexity (Simon \& Schuster, New York, 1993).

Gell-Mann, M. Europhys. News 33, 17 (2002). www.comdig.org

Nature Insight, Complex Systems Nature 410, 241-284 (2001). 